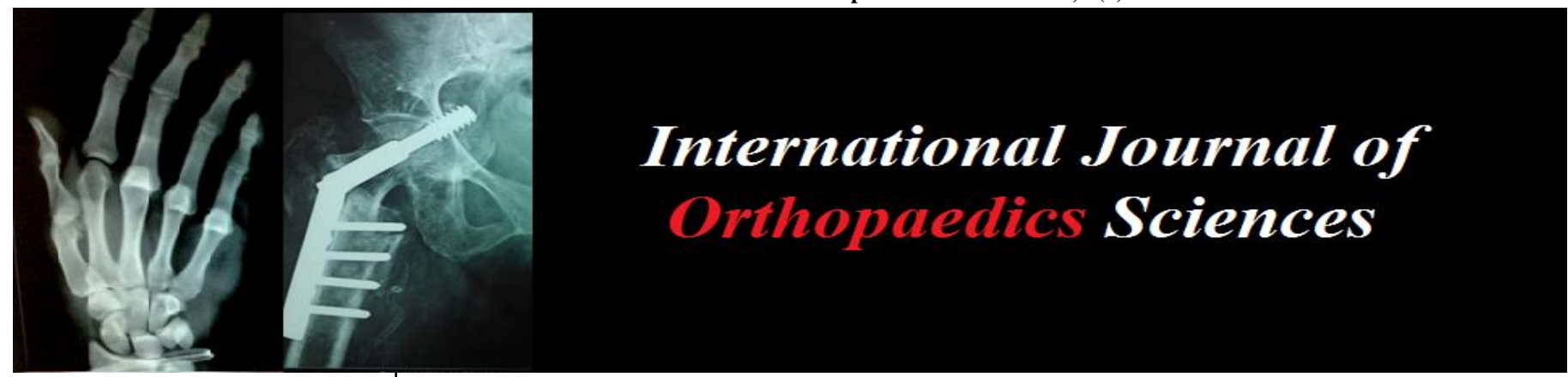

ISSN: $2395-1958$

IJOS 2018; 4(4): 06-10

(C) 2018 IJOS

www.orthopaper.com

Received: 03-08-2018

Accepted: 05-09-2018

Vishnu Sankar A

Assistant Professor, Department of orthopaedic Surgery, Govt

Thiruvannamalai medical college, Thiruvannamalai, Tamil Nadu, India

\section{Karthik R}

Senior Resident, Department of orthopaedic Surgery, PSG IMSR, Coimbatore, Tamil Nadu, India

\section{Arvind Manoj KK}

Assistant Professor, Department of orthopaedic surgery, Govt Sivagangai medical college, Sivagangai, Tamil Nadu, India

\section{Correspondence}

Karthik R

Senior Resident, Department of orthopaedic Surgery, PSG IMSR, Coimbatore, Tamil Nadu, India

\section{Critical analysis of prognostic factors in fracture dislocation of hip}

\author{
Vishnu Sankar A, Karthik R and Arvind Manoj KK
}

DOI: https://doi.org/10.22271/ortho.2018.v4.i4a.02

\section{Abstract}

Background: Fracture dislocation of the hip is an absolute orthopaedic emergency which is increasing day by day. There are various factors determining the functional outcome in these patients. The aim of the study is to critically analyse various prognostic factors and effect of acetabular fracture index (AFI), posterior acetabular arc angle (PAAA), head wall index (HWI) on functional outcome as well as importance of timing of relocation in development of degenerative arthritis.

Materials and methods: It is a prospective study including 23 patients, above 20 years of age with study period from January 2015 to May 2018. All the patients were evaluated clinically using modified Merle d` Aubigne Postel score and radiologically by Matta et al. criteria. The average follow up period was $2 \frac{1 / 2}{1}$ years.

Results: $48 \%$ of cases had excellent outcome, $31 \%$ of cases had good and $13 \%$ of cases had fair outcome. $8 \%$ of cases end with poor outcome. The quality of reduction was anatomical in $61 \%$ of cases and imperfect in $30 \%$ of cases and poor in $9 \%$ of cases. The functional outcome was good in patients with higher value of acetabular fracture index, posterior acetabular arc angle and lesser head wall index. Conclusion: The quality of reduction of fracture remains the most important factor when compared to AFI, PAAA, HWI in determining the functional outcome. The timing of relocation of hip may be of less significance in fracture dislocation.

Keywords: acetabular fracture index (AFI), posterior acetabular arc angle (PAAA), head wall index (HWI), fracture dislocation

\section{Introduction}

Traumatic dislocation and fracture dislocation of hip is an absolute orthopedic emergency that is steadily increasing in incidence. Early recognition of these fractures, and prompt, stable reduction is the essence of successful management ${ }^{[1]}$. Several authors have recommended urgent reduction of the dislocated hip in fracture dislocation as one of the most important factors to avoid the complications of secondary arthritis, avascular necrosis, but there is a lack of agreement with respect to the allowable timing, which ranges from less than six hours to within 24 hours ${ }^{[1-7]}$.

Apart from timing of relocation, various factors like the quality of reduction of the fracture, severity of injury, the age of the patient, damage to the femoral head, incarcerated fracture fragments, associated injuries, acetabular fracture index, posterior acetabular arc angle, head wall index, acetabular impaction, timing of surgery and the type of fracture plays a role in determining the functional outcome. There are very few studies in the literature suggesting the importance of tomographic parameters like acetabular fracture index, posterior acetabular arc angle, head wall index as predictors of functional outcome. So the aim of our study was to critically analyses these parameters, and determine the importance of time of relocation in determining the incidence of avascular necrosis and degenerative arthritis.

\section{Materials and method}

This is a prospective study conducted in Government Royapettah hospital, Chennai from January 2015 to May 2018. After institutional ethical committee clearance, 23 patients (21 male, 2 female) with age more than 20 years who sustained dislocation of hip with associated acetabulum fracture of duration less than 2 weeks were included in the study. Patients with Patients with fracture duration more than 2 weeks, or with associated degenerative arthritis 
changes in the hip joint were excluded. The mean age of the study population was 42 years and the mode of injury was road traffic accident in 18 patients $(78 \%)$ and fall from height in 5 patients $(22 \%)$. Majority of patients (20 cases) presented with posterior dislocation and 3 patients with central dislocation. The average time of presentation was 8 hours (range 2 hour- 26 hours).The associated injuries were distal radius fracture in 3 patients $(13 \%)$, proximal tibial fracture in 2 patients $(9 \%)$, ankle injury in 2 patients $(9 \%)$, fracture both bone leg in 1 patient (4\%) and radial head fracture in 1 patient (4\%). The acetabulum fractures were classified as per Judet and Letournel classification system into elementary and associated fractures ${ }^{[14]}$. 13 patients $(56 \%)$ presented with posterior wall fractures, 5 patients $(22 \%)$ with posterior wall and posterior column fractures and 5 patients (22\%) with transverse and posterior wall fracture. Pre-operative CT scan was done in all patients to study the tomographic parameters like acetabular fracture index (AFI), posterior acetabular arc angle (PAAA), head wall index (HWI).

The acetabular fracture index (AFI) as per the literature, is defined as the percentage of remaining intact posterior acetabular arc in the fractured side in comparison to the normal side. ${ }^{[8]}$ It is described by the formula AFI= (angle alpha/ angle beta)*100, where alpha is the angle formed by the arc line to the remaining intact posterior acetabular margin on the fractured side, and beta is the angle formed by the arc line to the posterior acetabular margin on the contralateral normal side (Figure 1). It is useful for preoperative and intraoperative evaluation where open reduction and internal fixation is needed and for predicting the prognosis of a posterior wall acetabular fracture ${ }^{[16]}$.

Posterior acetabular arc angle (PAAA) measurement involves axial CT section at the thinnest medial wall and widest acetabulum. The posterior acetabular arc angle of the femoral head was measured by using the acetabular centre as the apex of the angle and it includes both the posterior half medial wall and posterior acetabular wall (Figure 2). It is a quantitative measurement and represents the amount of posterior acetabular coverage of the femoral head on the fractured side and does not require the normal side measurement as reference value ${ }^{[9]}$.

Head wall index (HWI) measurement involves axial CT slides with largest anteroposterior femoral head diameter on the fractured side. Point A corresponds to the translation between articular surface and the acetabular fossa on the anterior acetabular surface; point B corresponds to the transition between the articular surface and the acetabular fossa on posterior acetabular surface; the third point (PW) corresponds to the posterior articular edge on the fractured side; the fourth point $(\mathrm{CH})$ corresponds to the femoral head center. The baseline was drawn through $\mathrm{A}$ and $\mathrm{B}$ points, and two perpendicular lines drawn to the BL: $\mathrm{W}$ line from the BL: and $\mathrm{H}$ line from the lateral edge of the femoral head, passing through $\mathrm{CH}$ to $\mathrm{BL}$ (Figure 3). The ratio between $\mathrm{H}$ and $\mathrm{W}$ is defined as head wall index ${ }^{[10]}$. The timing of relocation was calculated as the time interval between the injury time and reduction of the joint

The mean duration from fracture dislocation to surgery was 4 days with range from 2 to 12 days. Posterior wall and column fracture was addressed by Kocher Langenbech approach and anterior wall and column fracture by Stoppa approach. Postoperative anteroposterior (AP) view and Judet view (iliac oblique and obturator oblique) were taken to assess the quality of reduction as anatomical ( 0 to $1 \mathrm{~mm}$ displacement), imperfect ( 2 to $3 \mathrm{~mm}$ displacement) and poor (> $3 \mathrm{~mm}$ displacement) as per Matta et al criteria. ${ }^{[1,12]}$ Radiological outcome was assessed using Matta radiological outcome grading as excellent (normal joint space and no osteophytes and sclerosis), good (>1 mm joint space narrowing with small osteophytes and minimal sclerosis), fair $(<50 \%$ joint space narrowing with moderate osteophytes and sclerosis) and poor (>50\% joint space narrowing with large osteophytes and severe sclerosis) ${ }^{[12,13]}$. Mean follow up period was $2 \frac{1}{2}$ years, ranging from 2 years to $3 \frac{1}{2}$ years. Post-operative complications included 2 patients with superficial wound infection, 1 patient with primary sciatic nerve injury, 1 patient with heterotrophic ossification.

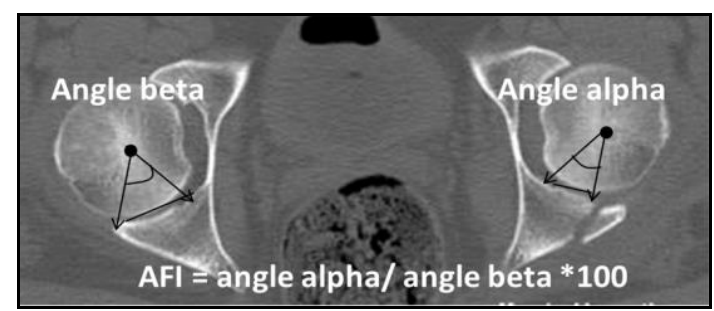

Fig 1: Measurement of acetabular fracture index

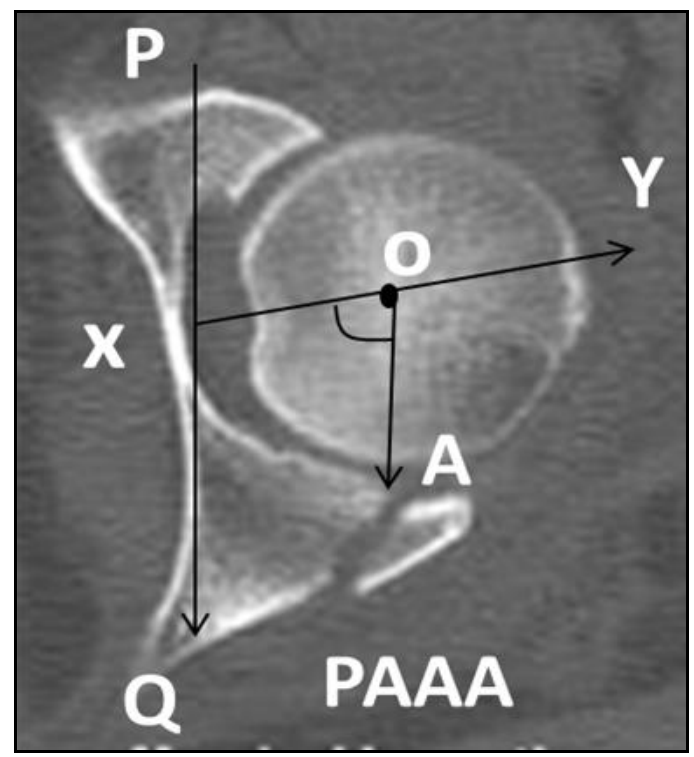

Fig 2: Measurement of Posterior acetabular arc angle (PAAA)

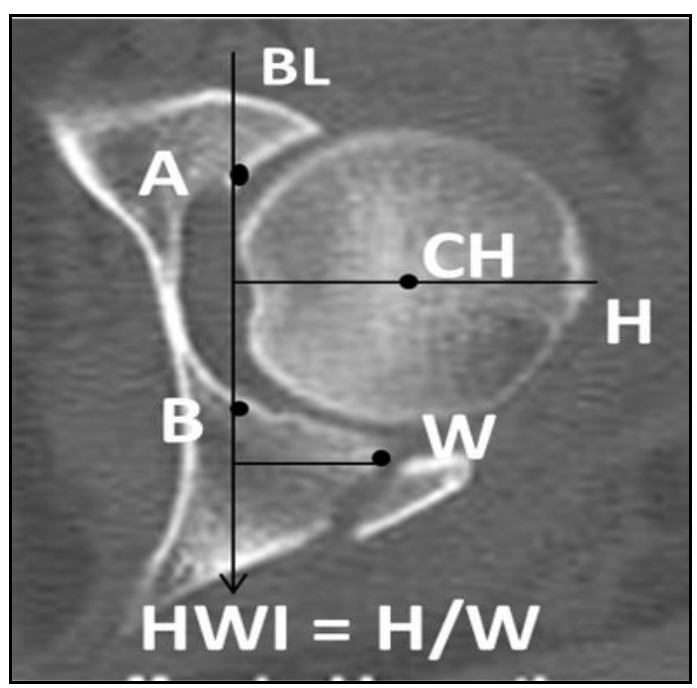

Fig 3: Measurement of head wall index

\section{Statistical analysis}

The statistical analysis in our study was performed using 
SPSS software version 20.0 and the various factors influencing the functional outcome like quality of reduction, AFI, PAAA, HWI and timing of relocation were analysed using Pearson Chi-Square analysis. All data were analysed at a significance level of $\mathrm{P}<0.05$.

\section{Results}

The mean modified Merle d Aubigne Postel score was 15.5, 11 patients $(48 \%)$ had excellent outcome (Figure 4), 7 patients (31\%) had good and 3 patients (13\%) had fair outcome. 2 patients $(8 \%)$ ended with poor outcome. We had one patient with secondary arthritic changes at 1 year follow up who was operated on $12^{\text {th }}$ day due to late presentation after initial trauma were the quality of reduction was poor (Figure 5)

From our study we found that the functional outcome was comparatively good in patients with anatomical reduction than in patients with imperfect or poor reduction (Table 1). The quality of reduction significantly affected the functional outcome $(\mathrm{P}=0.002)$. Also tomographic parameters life AFI, PAAA, HWI significantly affected the final functional outcome. Patient with high acetabular fracture index and posterior acetabular arc angle values had good functional outcome whereas patients with low head wall index value had good functional outcome. In our study the timing of relocation had no clinical significance with the functional outcome of the patients $(\mathrm{P}=0.371)$. (Table 2$)$
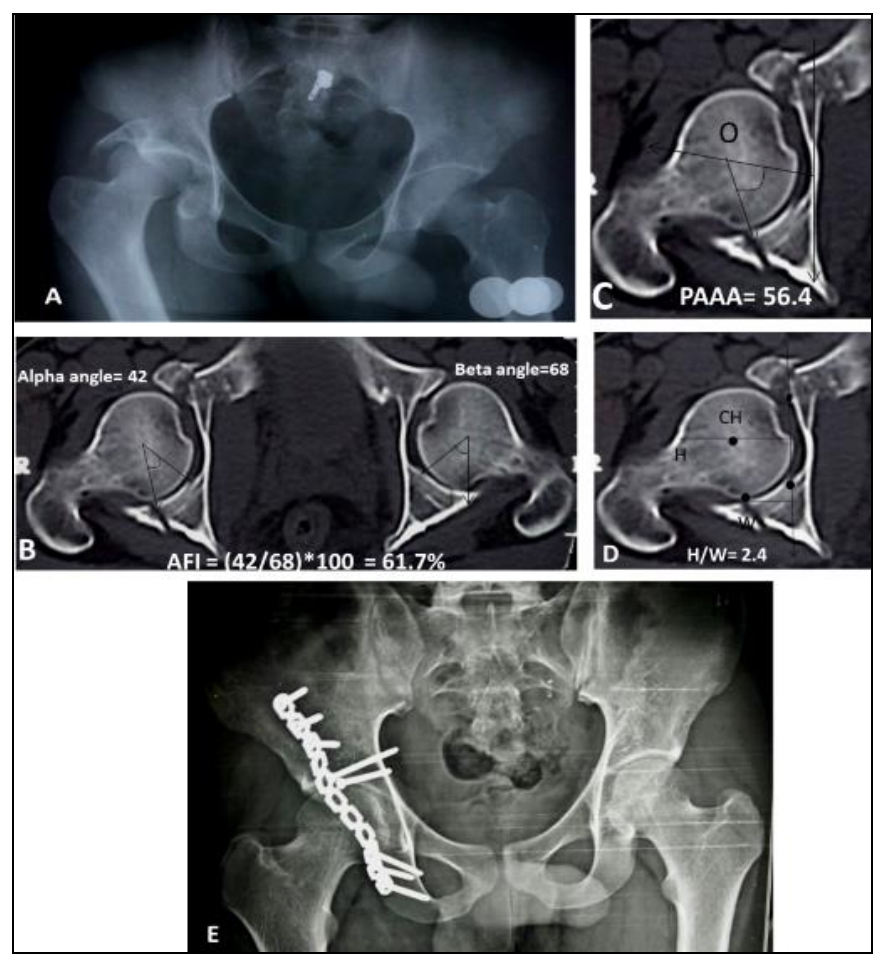

Fig 4: 51 year's old male with posterior dislocation and posterior wall fracture of right acetabulum A) preoperative x -ray, B) AFI measurement,

C) PAAA measurement, D) HWI measurement, E) 3 years follow up x ray
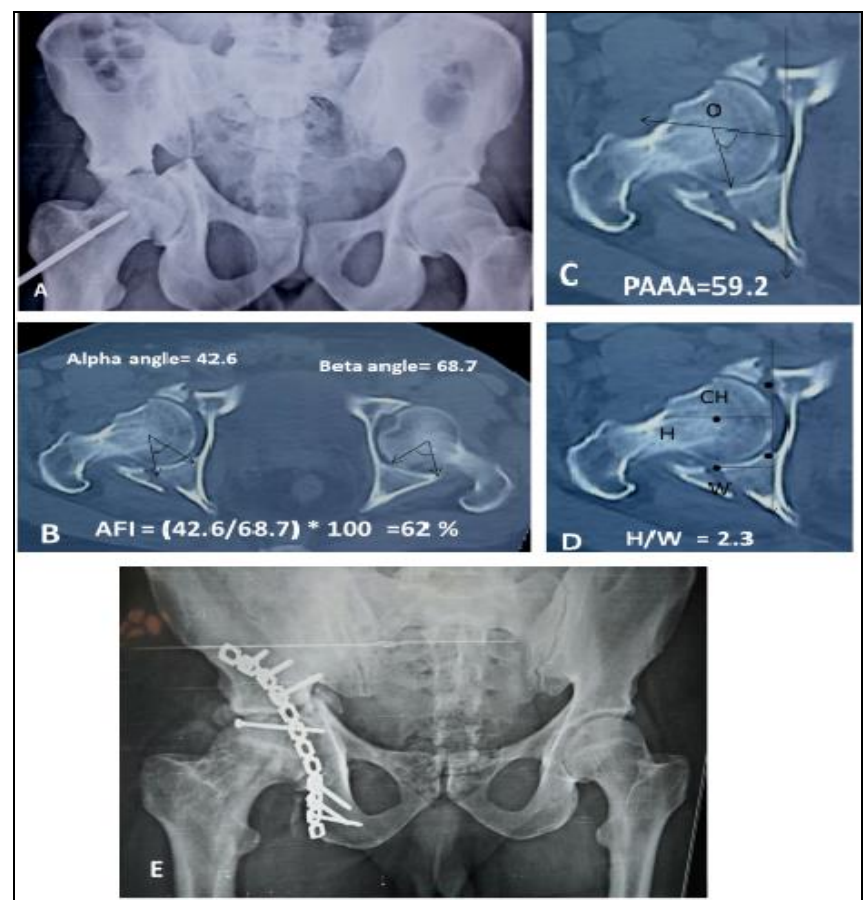

Fig 5: 58 year old male with central dislocation with transverse with posterior wall fracture A) Preoperative X-ray, B) measurement of AFI, C) measurement of PAAA, D) measurement of HWI, E) 1 year follow up x-ray with secondary arthritis due to poor reduction 
Table 1: Comparison of quality of reduction with clinical and radiological outcome

\begin{tabular}{|c|c|c|c|c|}
\hline \multirow{2}{*}{ Reduction quality } & \multicolumn{2}{|c|}{ Modified Merle d Aubigne Postel score } & \multicolumn{2}{c|}{ Matta radiological outcome grading } \\
\cline { 2 - 5 } & Excellent + good & Fair + poor & Excellent + good & Fair+ poor \\
\hline Anatomical & $14(61 \%)$ & - & $14(61 \%)$ & - \\
\hline Imperfect & $4(17 \%)$ & $3(13 \%)$ & $3(13 \%)$ & $4(17 \%)$ \\
\hline Poor & - & $2(9 \%)$ & - & $2(9 \%)$ \\
\hline Total & $18(78 \%)$ & $5(22 \%)$ & $17(74 \%)$ & $6(26 \%)$ \\
\hline
\end{tabular}

Table 2: Comparison of significance of various factors on functional outcome

\begin{tabular}{|c|c|c|c|c|c|c|}
\hline \multirow{2}{*}{ Factors } & \multicolumn{5}{|c|}{ Functional Outcome } & \multirow{2}{*}{ P-value } \\
\hline & \multicolumn{2}{|c|}{ Excellent \& Good } & \multicolumn{2}{|r|}{ Fair \& Poor } & Total & \\
\hline \multicolumn{7}{|c|}{ Quality of Reduction } \\
\hline Anatomical & 14 & $100.00 \%$ & 0 & $0.00 \%$ & 14 & 0.002 \\
\hline Imperfect & 4 & $57.14 \%$ & 3 & $42.86 \%$ & 7 & \\
\hline Poor & 0 & $0.00 \%$ & 2 & $100.00 \%$ & 2 & \\
\hline \multicolumn{7}{|l|}{ Relocation Time } \\
\hline$<6$ hours & 10 & $90.91 \%$ & 1 & $9.09 \%$ & 11 & 0.371 \\
\hline $6-12$ hours & 4 & $66.67 \%$ & 2 & $33.33 \%$ & 6 & \\
\hline$>12$ hours & 4 & $66.67 \%$ & 2 & $33.33 \%$ & 6 & \\
\hline \multicolumn{7}{|c|}{ AFI } \\
\hline$<25 \%$ & 0 & $0.00 \%$ & 1 & $100.00 \%$ & 1 & 0.015 \\
\hline $25 \%-50 \%$ & 2 & $40.00 \%$ & 3 & $60.00 \%$ & 5 & \\
\hline $50 \%-75 \%$ & 15 & $93.75 \%$ & 1 & $6.25 \%$ & 16 & \\
\hline$>75 \%$ & 1 & $100.00 \%$ & 0 & $0.00 \%$ & 1 & \\
\hline \multicolumn{7}{|c|}{ PAAA } \\
\hline$<50$ degree & 2 & $33.33 \%$ & 4 & $66.67 \%$ & 6 & 0.008 \\
\hline 50 - 70 degree & 15 & $93.75 \%$ & 1 & $6.25 \%$ & 16 & \\
\hline$>70$ degree & 1 & $100.00 \%$ & 0 & $0.00 \%$ & 1 & \\
\hline \multicolumn{7}{|c|}{ HWI } \\
\hline$<2.5$ & 5 & $83.33 \%$ & 1 & $16.67 \%$ & 6 & 0.042 \\
\hline $2.5-5.0$ & 9 & $100.00 \%$ & 0 & $0.00 \%$ & 9 & \\
\hline$>5.0$ & 4 & $50.00 \%$ & 4 & $50.00 \%$ & 8 & \\
\hline
\end{tabular}

\section{Discussion}

Fracture dislocation of hip resulting from high velocity trauma always had resulted in complex acetabular fractures which makes their management a tedious task for the operating surgeon. To improve the overall results in these patients, the need for understanding the various factors determing the functional and radiological outcome of these complex fractures gains prime importance. Many factors determine the functional outcome, which are categorised in literature as surgeon independent factors and surgeon dependent factors ${ }^{[15]}$.

The factors which are considered as surgeon independent factors includes patient age, gender, comorbidities, mechanism of injury, velocity of trauma, type of dislocation, type of acetabulum fracture, associated injuries, primary sciatic nerve damage etc. Whereas other factors like timing of surgery, duration of surgery, surgical blood loss, quality of reduction, iatrogenic sciatic nerve injury, post-operative mobilisation are within surgeon control.

In various literature, the quality of reduction which is nothing but restoration of articular congruity especially in the weight bearing dome is determined as the most important predictive variable of functional outcome and development of secondary arthritis in acetabular fractures ${ }^{[11-15]}$. Similarly in our sudy also, patients with anatomical reduction $(61 \%)$ had better functional outcome than patients with imperfect $(30 \%)$ or poor reduction (9\%). Inspite of near anatomical reduction, results had been poor in patients with marginal impaction where the internal fixation becomes unstable in post-operative period. These impacted fragments are generally raised and the void in the metaphyseal region is filled with cancellous bone graft followed by rigid fixation to achieve articular congruity. In posterior dislocation of hip with associated posterior wall fracture, the stability of reduction is determined by the size and number of posterior wall fragments and the remaining intact posterior wall. Several methods of measuring the posterior wall fragment were described in literature. Calkins et al in his study used a plastic template to mark the center of the acetabular arc and a goniometer to measure the acetabular fracture index approximately ${ }^{[16]}$. In our study we used CT PACS software to confirm the center of the acetabular arc and measure acetabular fracture index more precisely. Calkins et al. found that all hips with $<34 \%$ of the remaining posterior acetabulum were unstable and requires surgical intervention and that hips with $>55 \%$ were stable and had better outcome. ${ }^{[16]}$ Vailas et al. reported that fragments involving $25 \%$ of the acetabulum were stable, while fragments involving $50 \%$ can affect joint stability. ${ }^{[17]}$ In our study, patients with greater AFI had better functional outcome similar to study by Hai-Tao Long et al $^{[8]}$.

Similar to measurement of AFI, posterior acetabular arc angle was measured using CT coronal section without using the normal contralateral acetabulum as the calculation reference. In contrast to the AFI, PAAA is measured by taking the acetabular centre as the apex of angle instead of femoral head centre. Harnroongroj et al in their study concluded the stability of posterior fracture dislocation as stable and unstable based on PAAA value of $>70$ degree and $<50$ degree respectively ${ }^{[9]}$. In your study we analysed the PAAA with the functional outcome and found that patient with PAAA greater than 50 degree had better functional outcome.

Head wall index is another tomographic index described in literature to assess the stability of hip following fracture dislocation. Fujiki et al in their study made a clear cut description of head wall index and finally concluded that head wall index value of 2.4 as auxiliary criteria indicating the 
stability of hip ${ }^{[10]}$. In our study, we studied the significance of HWI with the functional outcome and found that patients with HWI less than 5 had better outcome than patients with HWI more than 5 .

Apart from these tomographic parameters, factors like fracture type, degree of fracture displacement, timing of surgery, associated injuries, duration of follow up, physical therapy had been already studied by various authors. ${ }^{[13,18,19]}$ In the study by Meena et al, comprising of 118 surgically treated acetabular fractures, the functional outcome was significantly affected by the quality of reduction, presence of associated injuries, initial fracture displacement, joint dislocation and delay in surgery ${ }^{[20]}$. Regarding the timing of relocation, there always had been controversy among various authors. Some authors suggests relocation timing of less than 12 hours associated with better functional outcome ${ }^{[21]}$. Hougaard et al in their study comprising of 98 patients with 100 posterior dislocations of the hip, the interval between injury and reduction was reported as a prognostic factor for clinical outcome, with hips reduced within six hours having a good or excellent result, compared with hips reduced after more than six hours ${ }^{[22]}$. But the results in our study were similar to Letournel and Judet study ${ }^{[14]}$ Bhandari et al study ${ }^{[23]}$, were the time interval between injury and reduction had no significant influence over the functional outcome and onset of secondary arthritis. Overall rate of AVN of $7.5 \%$ in their study population was observed by Letournel and Judet ${ }^{[14]}$. but no case of AVN has been reported so far in our study with average follow up of 2 years.

From our study, we conclude that the quality of reduction is the single most important factor determining the functional and radiological outcome in patients with fracture dislocation of hip. Tomographic parameters like AFI, PAAA, HWI may play a crucial role in determining the outcome. But the timing of relocation in fracture dislocation cases may not be as significant as in cases with pure dislocation as long as anatomical reduction is achieved.

\section{Declarations}

Funding: Nil

Conflict of interest: Nil

Ethical approval: the study was approved by institutional ethical committee.

\section{References}

1. Sahin V, Karakas ES, Aku Sa, et al. Traumatic dislocation and fracture-dislocation of the hip: a long-term follow-up study. J Trauma. 2003; 54:520-9.

2. Alonge TO, Ogunlade SO, Idowu OE. Traumatic dislocation of the hip joint: pattern and management in a tropical African population. West Afr J Med. 2002; 21:288-90.

3. Yang RS, Tsuang YH, Hang YS, Liu TK. Traumatic dislocation of the hip. Clin Orthop. 1991; 265:218-27. Jacob JP, Rao JP, Ciccarelli C. Traumatic dislocation and fracture dislocation of the hip: a long-term follow-up study. Clin Orthop. 1987; 214:249-63.

4. Jacob JP, Rao JP, Ciccarelli C. Traumatic dislocation and fracture dislocation of the hip: a long-term follow-up study. Clin Orthop. 1987; 214:249-63.

5. Pape HC, Rice J, Wolfram K, et al. Hip dislocation in patients with multiple injuries: a followup investigation. Clin Orthop. 2000; 377:99-105.

6. Hougaard K, Thomsen PB. Coxarthrosis following traumatic posterior dislocation of the hip. J Bone Joint
Surg [Am]. 1987; 69:679-83.

7. Reigstad A. Traumatic dislocation of the hip. J Trauma 1980; 20:603-6.

8. Long HT, Deng ZH, Zou M, Lin ZY, Zhu JX, Zhu Y. Effects of the acetabular fracture index and other factors of posterior wall acetabular fracture on functional outcome. J Int Med Res. 2017; 45(4):1394-1405.

9. Harnroongroj $T$, Suangyanon $P$, Tharmviboonsri $T$, Harnroongroj T. Posterior acetabular arc angle of the femoral head assesses instability of posterior fracturedislocation of the hip. Int Orthop. 2013; 37(6):1141-1145.

10. Fujiki EN, Yamaguchi EN, Miachiro E, Chikude T, Ikemoto RY, de Abreu LC, et al. Tomographic index as auxiliary criteria for surgery indication in fracture dislocation of acetabulum posterior wall. Int Arch Med. 2012; 5:18.

11. Matta JM. Fractures of the acetabulum: accuracy of reduction and clinical results in patients managed operatively within three weeks after the injury. J Bone Joint Surg Am. 1996; 78(11):1632-1645.

12. Matta JM. Operative treatment of acetabular fractures through the ilioinguinal approach: a 10 year perspective. Clin Orthop. 1994; 305:10-19.

13. Matta JM, Mehne DK, Roffi R. Fractures of the acetabulum: early results of a prospective study. Clin Orthop Relat Res. 1986; (205):241-250.

14. Letournel E, Judet R. Fractures of the acetabulum. Elson RA, ed. New York: Springer-Verlag, 1993.

15. Briffa N, Pearce R, Hill AM, Bircher M. Outcomes of acetabular fracture fixation with ten years' follow-up. J Bone Joint Surg Br. 2011; 93(2):229-236.

16. Calkins MS, Zych G, Latta L, Borja FJ, Mnaymneh W. Computed tomography evaluation of stability in posterior fracture dislocation of the hip. Clin Orthop Relat Res 1988; 227:152-163.

17. Vailas JC, Hurwitz S, Wiesel SW. Posterior acetabular fracture-dislocations: fragment size, joint capsule, and stability. J Trauma 1989; 29:1494-1496.

18. Moed BR, Yu PH, Gruson KI. Functional outcomes of acetabular fractures. J Bone Joint Surg Am. 2003; 85(10):1879-1883.

19. Murphy D, Kaliszer M, Rice J, McElwain JP. Outcome after acetabular fracture. Prognostic factors and their interrelationships. Injury. 2003; 34(7):512-517.

20. Meena UK, Tripathy SK, Sen RK, Aggarwal S, Behera P. Predictors of postoperative outcome for acetabular fractures. Orthop Traumatol Surg Res. 2013; 99(8):929935.

21. Moed BR, Willson Carr SE, Watson JT. Results of operative treatment of fractures of the posterior wall of the acetabulum. J Bone Joint Surg [Am]. 2002; 84:752-8.

22. Hougaard K, Thomsen PB. Coxarthrosis following traumatic posterior dislocation of the hip. J Bone Joint Surg [Am] 1987; 69:679-83.

23. Bhandari M, Matta J, Ferguson T, Matthys G. Predictors of clinical and radiological outcome in patients with fractures of the acetabulum and concomitant posterior dislocation of the hip. J Bone Joint Surg Br. 2006; 88:1618-1624. 